\title{
OPERATIONAL PERFORMANCE OF A BUNCH BY BUNCH DIGITAL DAMPER IN THE FERMILAB MAIN INJECTOR*
}

\author{
P. Adamson ${ }^{\dagger \ddagger}$, W. J. Ashmanskas, G. W. Foster, S. Hansen, A. Marchionni, \\ D. Nicklaus, A. Semenov, D. Wildman, Fermilab, Batavia, Illinois \\ H. Kang, Stanford University, Stanford, California
}

\section{Abstract}

We have implemented a transverse and longitudinal bunch by bunch digital damper system in the Fermilab Main Injector, using a single digital board for all 3 coordinates. The system has been commissioned over the last year, and is now operational in all MI cycles, damping beam bunched at both $53 \mathrm{MHz}$ and $2.5 \mathrm{MHz}$. We describe the performance of this system both for collider operations and high-intensity running for the NuMI project.

\section{INTRODUCTION}

The Main Injector is the nexus at the heart of Fermilab's accelerator complex. As such, it runs in a large number of different modes, with typically three or four arbitrary states present in a supercycle at any one time. The major operating states are listed in table 1 . Proton shots to the Tevatron coalesce typically $753 \mathrm{MHz}$ bunches, the antiproton source and the Switchyard fixed target area use 1 booster batch (84 bunches) and the NuMI (Main Injector neutrino beam) cycles usually use a full ring of 6 booster batches.

\begin{tabular}{|c|c|c|}
\hline protons & $53 \mathrm{MHz}$ & $\begin{array}{l}120 \mathrm{GeV} \text { beam to antipro- } \\
\text { ton source }\end{array}$ \\
\hline protons & $53 \mathrm{MHz}$ & $120 \mathrm{GeV}$ beam to NuMI \\
\hline protons & $53 \mathrm{MHz}$ & $\begin{array}{l}120 \mathrm{GeV} \text { beam to Switch- } \\
\text { yard }\end{array}$ \\
\hline protons & $53 \mathrm{MHz}$ & $\begin{array}{l}120 \mathrm{GeV} \text { beam to antipro- } \\
\text { ton source and NuMI }\end{array}$ \\
\hline protons & $53 \mathrm{MHz}$ & $\begin{array}{l}120 \mathrm{GeV} \text { beam to antipro- } \\
\text { ton source and Switchyard }\end{array}$ \\
\hline protons & $53 \mathrm{MHz}$ & $150 \mathrm{GeV}$ beam to Tevatron \\
\hline antiprotons & $\begin{array}{l}2.5 \mathrm{MHz} \\
\& 53 \mathrm{MHz}\end{array}$ & $\begin{array}{l}150 \mathrm{GeV} \text { beam from Accu- } \\
\text { mulator to Tevatron }\end{array}$ \\
\hline antiprotons & $\begin{array}{l}2.5 \mathrm{MHz} \\
\& 53 \mathrm{MHz}\end{array}$ & $\begin{array}{l}150 \mathrm{GeV} \text { beam from Recy- } \\
\text { cler to Tevatron }\end{array}$ \\
\hline antiprotons & $2.5 \mathrm{MHz}$ & $8 \mathrm{GeV}$ beam to Recycler \\
\hline
\end{tabular}

Table 1: Major operating states in the Main Injector

A damper system for the Main Injector needs to operate and be independently configured on all these different states, dealing with protons and antiprotons bunched in

\footnotetext{
* Work supported by Universities Research Association, Inc. under contract no. DE-AC02-76CH03000 with the U.S. Department of Energy.

${ }^{\dagger}$ pa@fnal.gov

$\ddagger$ also at Dept. of Physics and Astronomy, University College London, London WC1E 6BT, UK
}

both $53 \mathrm{MHz}(\mathrm{h}=588)$ and $2.5 \mathrm{MHz}(\mathrm{h}=28)$ buckets, sometimes operating with a full ring and sometimes with only a few buckets populated.

Building on the experience obtained with a prototype system[1], a digital transverse and longitudinal bunch-bybunch damper has been constructed and installed in the Main Injector.

\section{HARDWARE}

A schematic diagram of the damper hardware is shown in figure 1. For the transverse dampers, we use $1 \mathrm{~m}$ long stripline pickups and kickers inherited from the Main Ring. For the longitudinal damper, a resistive wall current monitor provides the input signal, and three purposebuilt broadband cavities, similar to those built for the Fermilab Recycler[2] but with improved high frequency response, provide the kick. The cavities are each driven by a $3.5 \mathrm{~kW}$ Amplifier Research model 3500A100 amplifier. The stripline kickers are each driven by a pair of $500 \mathrm{~W}$ Amplifier Research model 500A100A amplifiers. Both these amplifiers have a bandwidth of $10 \mathrm{kHz}$ to $100 \mathrm{MHz}$.

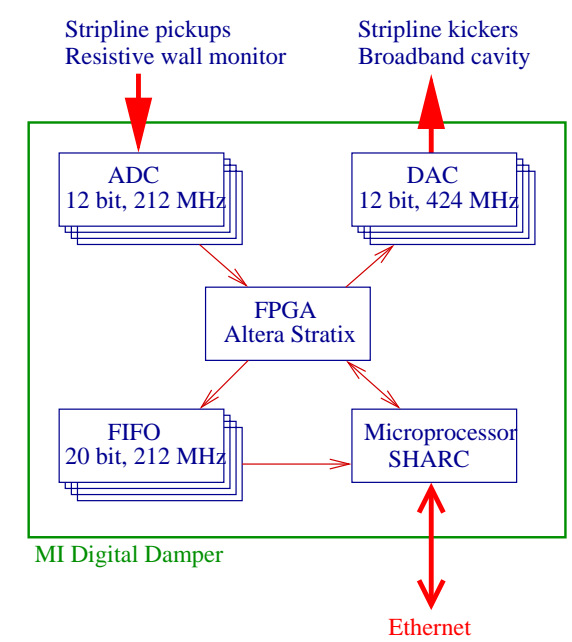

Figure 1: Schematic diagram of the main injector digital damper system.

The digital damper board itself contains a large field programmable gate array (Altera Stratix EP1S25) to do all the damping calculations, and a SHARC 21065 DSP processor abused to provide TCP/IP communication with the outside world. The signals from the pickups are smoothed 
by $70 \mathrm{MHz}$ lowpass filters, and sampled by 12-bit ADCs clocked at $212 \mathrm{MHz}$ (4 times the RF frequency). The board contains 4 FIFOs, each of 256k 20-bit words, clocked at $212 \mathrm{MHz}$, to support the capture and display of the input signals and quantities calculated in the FPGA. The output waveforms are synthesized by 12-bit DACs clocked at $424 \mathrm{MHz}$. The use of a rate 8 times the bunch frequency allows fine-tuning of the output waveform to correct for defects in the power amplifiers.

The damper is configured and controlled via its TCP/IP connection. New programs for the SHARC processor and the FPGA can be loaded in this way, and the per-state configuration of the damper is accessible via the standard controls system, ACNET. One may trivially change "live" which bunches are to be damped, the phase advance of the transverse FIR filter and so on.

\section{OPERATIONAL EXAMPLES}

The damper system has been used on all the operational modes mentioned earlier. We present here a few examples of its use.

\section{$53 \mathrm{MHz}$ longitudinal}

Longitudinal dampers at $53 \mathrm{MHz}$ are used on stacking, NuMI and proton coalescing cycles. They successfully reduce longitudinal emmitance for beam to the pbar source, and by controlling the longitudinal emmitance increase the efficiency of coalescing (see figure 2.)
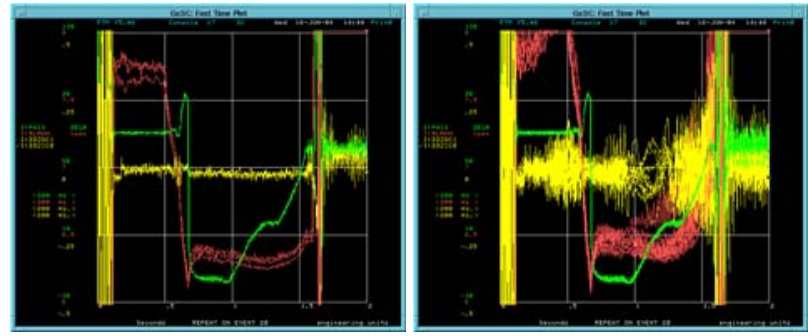

Figure 2: Longitudinal dampers on (left) and off (right) on a proton coalescing cycle. The yellow trace shows the longitudinal phase error of the central bunch.

\section{$53 \mathrm{MHz}$ transverse}

The need for transverse damping became acute with the introduction of the NuMI cycles. Without dampers we were able to control the resistive wall instability with large negative chromaticity, however this resulted in a poor lifetime at $8 \mathrm{GeV}$. We achieve a much better lifetime by using the transverse dampers to control the instability, and running with chromaticities close to 0 . Figure 3 shows the vertical oscillation of one bunch after injection. A $1 \mathrm{~mm}$ oscillation is damped to nothing in about 40 machine turns.

Figure 4 shows the long $8 \mathrm{GeV}$ lifetime achieved with dampers and low chromaticity. Without the dampers, we

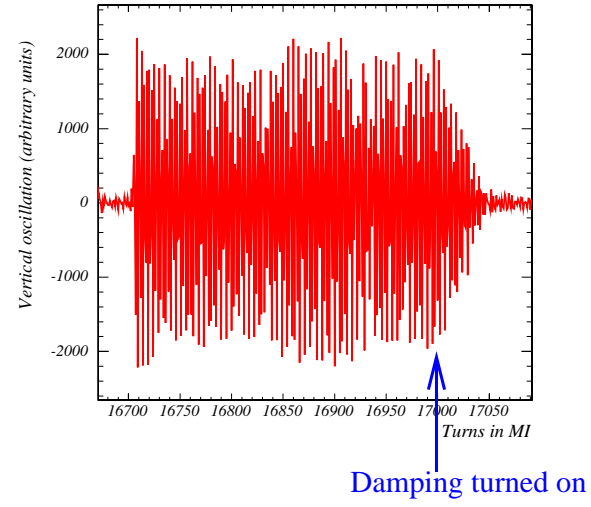

Figure 3: Damping of vertical injection oscillations. The initial oscillation amplitude is about $1 \mathrm{~mm}$.

would lose more than $10 \%$ of the beam during the $0.5 \mathrm{sec}$ ond $8 \mathrm{GeV}$ time.

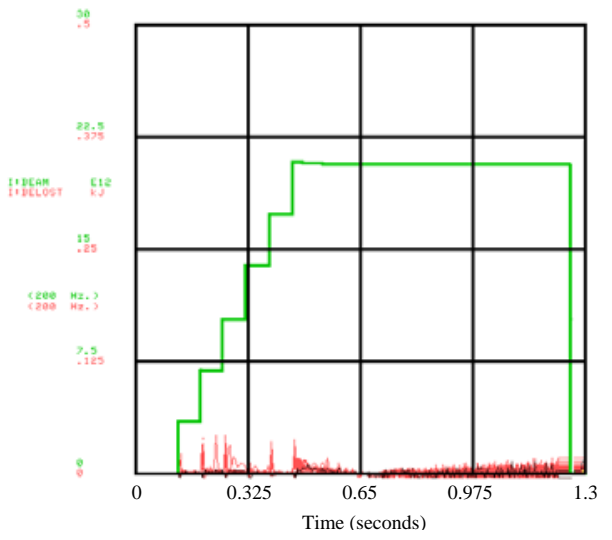

Figure 4: Transverse dampers allow long $8 \mathrm{GeV}$ lifetime, essential for multibatch injection on NuMI cycle. The green traces show the beam current.

\section{Antiproton shots to the Tevatron}

Antiprotons are transferred to the Main Injector from the Accumulator and Recycler at $8 \mathrm{GeV}$ in four $2.5 \mathrm{MHz}$ buckets. The buckets are compressed in the Main Injector before the beam is captured in $53 \mathrm{MHz}$ buckets for acceleration to $150 \mathrm{GeV}$, where it is coalesced into one $53 \mathrm{MHz}$ batch. At present, the beam is damped longitudinally at injection in $2.5 \mathrm{MHz}$ buckets, and again at $53 \mathrm{MHz}$ during acceleration before coalescing. Due to space constraints on the FPGA, we do not currently damp the coalesced bunch - it is hoped that a new version of the FPGA code will allow this.

Figure 5 shows the longitudinal distribution of the 4 $2.5 \mathrm{MHz}$ bunches at an early point in the development of the damper system. One can see longitudinal oscillations chiefly in the 2nd and 3rd bunches. The dampers were turned on about half a period after injection for the fourth bunch. 


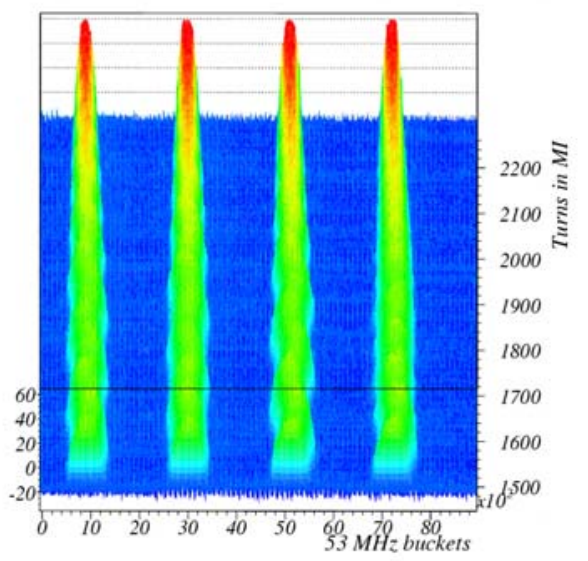

Figure 5: Longitudinal distribution of antiprotons bunched in $42.5 \mathrm{MHz}$ bunches. The fourth bunch is damped.

Figure 6 shows the bunch length of the coalesced antiproton bunches at $150 \mathrm{GeV}$ in the Main Injector, just prior to Tevatron injection, over the last few months. Although the data taken with the longitudinal dampers enabled do show a small reduction in bunch length (2.4 ns vs. 2.5 ns) the chief benefit of the damper system for antiproton shots is the reduction in tuning required. The damper system renders the machine significantly less sensitive to the incoming beam.

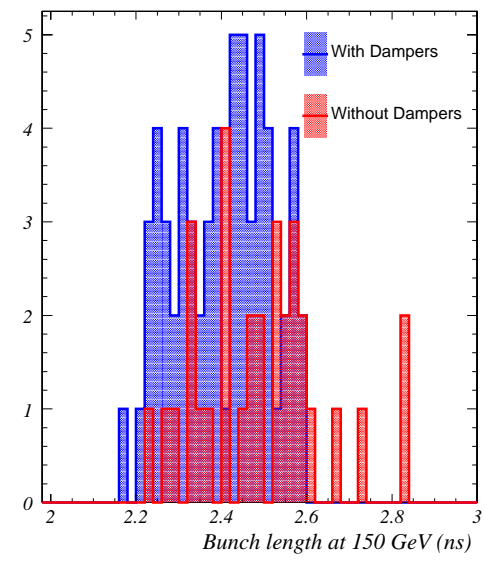

Figure 6: Bunch length at $150 \mathrm{GeV}$ after coalescing for antiprotons, before and after the introduction of longitudinal damping.

\section{DAMPER AS DIAGNOSTIC}

As mentioned earlier, the damper board includes $4256 \mathrm{k}$ word 20-bit FIFOs, clocked at the ADC sample rate of $212 \mathrm{MHz}$. This allows us to write out the raw ADC samples and the damping calculation products for all 3 coordinates. The memory is sufficient to store data from every bucket for 110 turns, one bucket for 65,000 turns, or anywhere in between. One can also skip an arbitrary number of turns between samples, thus extending our range in time to effectively infinity, albeit at a coarser sampling rate.

The inclusion of this fast FIFO on the damper board allows the damper system to provide useful diagnostic information. Figure 7 clearly shows a mode 1 instability, which occurs at high intensity on the NuMI cycles. The provision of such clear proof of what is going on makes it significantly easier to work towards a cure.

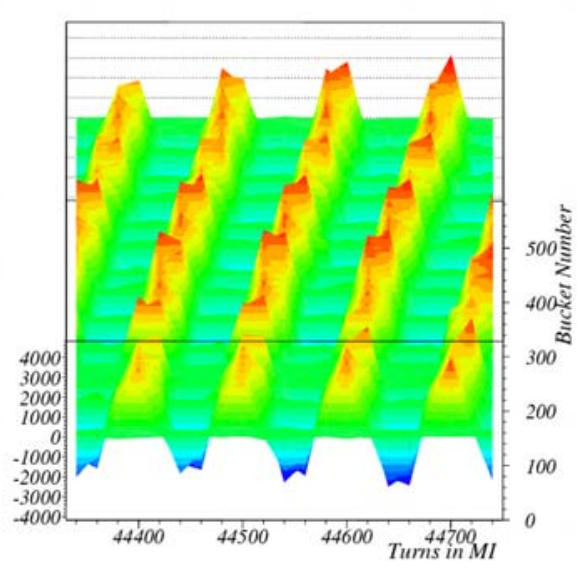

Figure 7: A coherent mode 1 instability on a NuMI study cycle. Plotted is the longitudinal phase error of each bunch, in arbitrary units, against turn and bunch number.

\section{FUTURE PLANS}

We have built a powerful and flexible bunch-by-bunch damper system, which also doubles as a useful and highly visual diagnostic tool. We are currently enhancing the firmware on the existing damper board in order to damp the mode 1 instability seen above. The broadband cavities, with a total maximum cavity voltage of $1.8 \mathrm{kV}$, do not offer enough power to damp this instability, so we will synthesize a $53 \mathrm{MHz}$ signal with slowly varying phase to add in to the main RF system.

Other plans include a next generation board, with a larger FPGA and a large RAM, to allow for greater flexibility and more powerful diagnostics, and further diagnostic tools using the existing hardware, such as a tune meter.

\section{REFERENCES}

[1] G. W. Foster et. al., "Bunch-by-Bunch Digital Dampers for the Fermilab Main Injector," PAC'03, May 2003, Volume 1 p. 323-325.

[2] G. Jackson, "The Fermilab recycler ring technical design report," Fermilab, November 1996. 